\title{
Incidence, aetiology and outcomes of obstetric-related acute kidney injury in Malawi: a prospective observational study
}

William R. Cooke ${ }^{1,2}$, Ulla K. Hemmilä ${ }^{1}$, Alison L. Craik¹, Chimwemwe J. Mandula', Priscilla Mvula ${ }^{3}$, Ausbert Msusa ${ }^{3}$, Gavin Dreyer ${ }^{5}$ and Rhys Evans ${ }^{1,4^{*}}$

\begin{abstract}
Background: Obstetric-related acute kidney injury (AKI) is thought to be a key contributor to the overall burden of AKI in low resource settings, causing significant and preventable morbidity and mortality. However, epidemiological data to corroborate these hypotheses is sparse. This prospective observational study aims to determine the incidence, aetiology and maternal-fetal outcomes of obstetric-related AKI in Malawi.

Methods: Women greater than 20 weeks gestation or less than 6 weeks postpartum admitted to obstetric wards at a tertiary hospital in Blantyre, Malawi, and at high-risk of AKI were recruited between 21st September and 11th December 2015. All participants had serum creatinine tested at enrolment; those with creatinine above normal range (> $82 \mu \mathrm{mol} / \mathrm{L}$ ) underwent serial measurement, investigations to determine cause of kidney injury, and were managed by obstetric and nephrology teams. AKI was diagnosed and staged by Kidney Disease Improving Global Outcomes (KDIGO) criteria. Primary outcomes were the incidence proportion and aetiology of AKI. Secondary outcomes were in-hospital maternal mortality, need for dialysis, renal recovery and length of stay; in-hospital perinatal mortality, gestational age at delivery, birthweight and Apgar score.
\end{abstract}

Results: 354 patients were identified at risk of AKI from the approximate 2300 deliveries that occurred during the study period. Three hundred twenty-two were enrolled and 26 (8.1\%) had AKI (median age 27 years; HIV 3.9\%). The most common primary causes of AKI were preeclampsia/eclampsia $(n=19,73.1 \%)$, antepartum haemorrhage $(n=3,11.5 \%)$, and sepsis $(n=3,11.5 \%)$. There was an association between preeclampsia spectrum and AKI (12.2\% AKI incidence in preeclampsia spectrum vs. $4.3 \%$ in other patients, $p=0.015)$. No women with AKI died or required dialysis and complete renal recovery occurred in 22 (84.6\%) cases. The perinatal mortality rate across all high-risk admissions was $13.8 \%$. AKI did not impact on maternal or fetal outcomes.

Conclusions: The incidence of AKI in high-risk obstetric admissions in Malawi is $8.1 \%$ and preeclampsia was the commonest cause. With tertiary nephrology and obstetric care the majority of AKI resolved with no effect on maternal-fetal outcomes. Maternal-fetal outcomes in Sub-Saharan Africa may be improved with earlier detection of hypertensive disease in pregnancy.

Keywords: Acute Kidney Injury, Acute renal failure, Global Health, Sub-Saharan Africa, Pregnancy, Preeclampsia

\footnotetext{
*Correspondence: rhysdrevans@gmail.com; rhys.evans@ucl.ac.uk

'Department of Internal Medicine, Queen Elizabeth Central Hospital, Blantyre,

Malawi

${ }^{4}$ University College London Centre for Nephrology, Royal Free Hospital, Pond

Street, London NW3 2QG, UK

Full list of author information is available at the end of the article
} 


\section{Background}

Acute Kidney Injury (AKI) in pregnancy causes significant morbidity and mortality $[1,2]$. It is increasingly recognised as a condition that is both preventable and treatable, often with simple and inexpensive interventions [3]. In developed countries, the incidence of AKI in pregnancy has been dramatically reduced as a result of improving clinical care [4]; a recent Canadian study found an AKI rate of 2.68 per 10,000 deliveries [5]. In developing countries, AKI in pregnancy is more common though less well studied, with AKI rates of 66 per 10,000 deliveries, for example, reported in Morocco [6].

The International Society of Nephrology recently launched the 0by25 initiative, which aims to eliminate preventable deaths from AKI by 2025 [7]. An initial goal is to establish the global burden of AKI, particularly in low-income settings. Over 50\% of global maternal deaths occur in Sub-Saharan Africa (SSA), but the contribution of kidney disease to these is unknown [8]. Only three studies investigating AKI in pregnancy from SSA have been published since 1990; these report AKI limited to dialysis units, intensive care and women with severe preeclampsia [9-11]. In these studies, the predominant causes of AKI were preeclampsia-eclampsia, haemorrhage and sepsis.

To the best of our knowledge, there are no previous studies investigating obstetric AKI outside intensive care and dialysis units in SSA; the causes and consequences of AKI for mother and fetus are unknown. This study aimed to determine the incidence proportion, aetiology and maternal-fetal outcomes of AKI in obstetric patients at high-risk for AKI admitted to a tertiary hospital in Malawi. In a population with a high fertility rate (mean 5 births per woman in SSA [12]) and high prevalence of preeclampsia, sepsis and haemorrhage [13], we predicted a high rate of obstetric-related AKI, leading to adverse outcomes for mother and fetus.

\section{Methods}

\section{Study setting and design}

We conducted a prospective observational study between 21st September and 11th December 2015 in the Obstetrics and Gynaecology Department of Queen Elizabeth Central Hospital (QECH), Blantyre, Malawi. This unit delivers approximately 12,000 women per year and receives tertiary referrals from across the southern region of the country, although the majority of admissions are from within Blantyre district itself.

\section{Inclusion and exclusion criteria}

All women aged 16 years and older, and greater than 20 weeks gestation or less than six weeks postpartum admitted to the Obstetric High Dependency Unit or highrisk areas of labour/antenatal/postnatal/gynaecology wards were assessed for risk of AKI within 48 h of admission. Presentation with one of 8 conditions was considered to place women at increased risk of AKI: gestational hypertension, preeclampsia, eclampsia, antepartum haemorrhage, postpartum haemorrhage, sepsis, heart failure and renal failure (Table 1). Patients with known renal failure were defined as a pre-existing diagnosis of CKD, or because a urea/creatinine result had already been obtained by the obstetric team and was elevated.

Study recruiters assessed whether AKI risk factor definitions were met; where insufficient information was documented to support or refute the presence of a risk factor, the treating obstetrician's clinical diagnosis was used. All women at increased risk of AKI were enrolled in the study, baseline clinical data were recorded, and screening serum creatinine ( $\mathrm{SCr}$ ) was measured to determine the presence/absence of kidney disease. Screening $\mathrm{SCr}>82 \mu \mathrm{mol} / \mathrm{L}$ was considered to be elevated, determined prospectively as being two standard deviations above mean SCr in the third trimester of pregnancy [14].

Women with elevated SCr on admission $(>82 \mu \mathrm{mol} / \mathrm{L})$ were followed up with daily measurement of $\mathrm{SCr}$ and urine output, in addition to detailed clinical assessment and management by both the nephrology and obstetric teams. We recorded the nature and most likely causes of renal impairment based on the assessment of all available clinical and laboratory investigations as well as details of management thereafter. Patients with normal $\mathrm{SCr}(<82 \mu \mathrm{mol} / \mathrm{L})$ at enrolment were managed by obstetricians and not routinely seen by the study team. The development of AKI in hospital was not assessed.

Maternal and fetal outcomes, identified from ward discharge and delivery records as well as individual patient records, were recorded in all women. Hospital maternal mortality records were crosschecked to ensure that no

Table 1 Study inclusion criteria and definitions

\begin{tabular}{ll}
\hline Inclusion criterion & Definition \\
\hline Gestational hypertension & $\begin{array}{l}\text { New onset hypertension after } 20 \text { weeks } \\
\text { gestation (defined as two BP readings }> \\
140 / 90 \text { separated by } \geq 4 \text { h) }\end{array}$ \\
Preeclampsia & $\begin{array}{l}\text { Gestational hypertension with dipstick } \\
\text { proteinuria } \geq 1+\end{array}$ \\
Eclampsia & $\begin{array}{l}\text { Seizure with preeclampsia } \\
\text { Sepsis }\end{array}$ \\
Antepartum haemorrhage & $\begin{array}{l}\text { Treating clinician's judgement } \\
\text { "spotting" }\end{array}$ \\
Postpartum haemorrhage & $\begin{array}{l}\text { Any documented bleeding considered by } \\
\text { the treating obstetrician not to be } \\
\text { physiological }\end{array}$ \\
Heart failure & Pre-existing clinical diagnosis \\
Renal failure & Pre-existing clinical diagnosis
\end{tabular}

Definitions for hypertensive diseases from American College of Obstetricians and Gynecologists [23] 
women lost to follow-up had died. Women were followed up until death, discharge, or a predefined period of 2 weeks after last recruitment.

\section{Creatinine measurement}

Serum creatinine ( $\mathrm{SCr}$ ) was tested (Jaffe method) using either a Flexor Junior Clinical Chemistry Analyser [Vital Scientific, The Netherlands] (31\% samples) or Mindray Chemistry Analyzer BS-120 [Shenzen Mindray BioMedical Electronics Company, China] (69\% samples). Biochemistry analysers were calibrated in accordance with the manufacturer's instructions. A second analyser was used due to unresolvable technical problems with the original machine during part of the study.

\section{Definitions}

AKI was diagnosed and staged according to Kidney Disease: Improving Global Outcomes (KDIGO) criteria [15] (Additional file 1). The lowest $\mathrm{SCr}$ recorded during admission or in the 3 months prior to admission was used as the baseline value. Imputed baselines based on a presumed normal Glomerular Filtration Rate (GFR) were not used in cases where the baseline was unknown.

Women with evidence of renal impairment $(\mathrm{SCr}>$ $82 \mu \mathrm{mol} / \mathrm{L}$ ) which was thought to be of less than 3 months duration but who did not fulfil KDIGO AKI criteria were categorised as having an Acute Kidney Disorder (AKD) and were not included in any further analysis. Women with renal impairment of greater than 3 months duration were diagnosed with Chronic Kidney Disease (CKD) as per KDIGO and were not included in any further analysis [15]. AKI aetiology was determined, after detailed clinical review of each case, by a panel of clinicians led by a nephrologist (UH).

Complete renal recovery was defined as final measured $\mathrm{SCr}<82 \mu \mathrm{mol} / \mathrm{L}$ after an initial SCr $>82 \mu \mathrm{mol} / \mathrm{L}$. Where mothers remained in hospital solely because their child was receiving neonatal care or were transferred to private wards, maternal length of stay data were considered to be unrepresentative of maternal outcomes and were excluded from the analysis.

Perinatal mortality was defined as fetal death after 20 weeks gestation (stillbirth or termination of pregnancy) or neonatal death before discharge from hospital (early or late neonatal death). Preeclampsia spectrum was defined as gestational hypertension, preeclampsia or eclampsia.

\section{Outcomes}

Primary outcome measures were the incidence proportion and aetiology of AKI. Secondary outcomes were maternal survival, need for maternal dialysis, renal recovery and maternal length of stay; fetal survival, gestational age at delivery, birthweight and Apgar score at $1 \mathrm{~min}$ after delivery [16].

\section{Statistical analyses}

We conducted a descriptive analysis of the study cohort. Variables are reported as mean \pm standard deviation (SD) or median \pm inter-quartile range (IQR) depending on their distribution. We compared variables between women with NKD and any stage of AKI using the Student's $t$ test if normally distributed, the Mann-Whitney $\mathrm{U}$ test if not normally distributed and Chi-squared or Fisher's exact test for proportions. Statistical analysis was performed using STATA version 10 (www.stata.com). A $p$ value of $<0.05$ was considered to represent statistical significance.

\section{Results}

Study recruitment is summarised in Fig. 1. Two thousand two hundred forty-six women delivered during the study recruitment period.

\section{Baseline data}

Demographic and baseline clinical data are reported in Table 2.

\section{Primary outcomes Incidence of $A K I$}

AKI of any stage was identified in 26 (8.1\%) recruited women (Fig. 1). 12 (46.2\%) cases of AKI were stage 1 [median screening SCr $99 \mu \mathrm{mol} / \mathrm{L}, \mathrm{IQR} 94$ - 118]; 8 (30.8\%) cases were stage 2 [median screening SCr $99 \mu \mathrm{mol} / \mathrm{L}, \mathrm{IQR}$ 96 - 120], and $6(23.1 \%)$ cases were stage 3 [median screening SCr $139 \mu \mathrm{mol} / \mathrm{L}, \mathrm{IQR} 135$ - 148].

\section{Aetiology of AKI}

The primary causes of AKI were preeclampsia/eclampsia $(n=19,73.1 \%)$, antepartum haemorrhage $(n=3,11.5 \%)$, sepsis $(n=3,11.5 \%)$ and cardiac failure $(n=1,3.8 \%)$. The sources of sepsis were endometritis, chorioamnionitis and Bartholin's abscess. In 13 (50\%) cases, multiple factors contributed to development of AKI; nephrotoxins (NSAIDs, tenofovir, gentamicin or traditional medications) were a contributing factor in 10 (38.5\%) cases.

\section{Secondary outcomes}

Maternal outcomes were determined in 254 (84.1\%) women. Two women died during their inpatient stay, neither had renal impairment. No woman required acute dialysis for AKI during her admission. Complete renal recovery occurred within 7 days in 22 (84.6\%) AKI cases overall ( $75 \%$ of stage $1,87.5 \%$ of stage 2 and $100 \%$ of stage $3 \mathrm{AKI})$. The remaining women $(n=4,15.4 \%)$ were discharged before their creatinine fell below $82 \mu \mathrm{mol} / \mathrm{L}$; three had evidence of partial renal recovery prior to discharge 


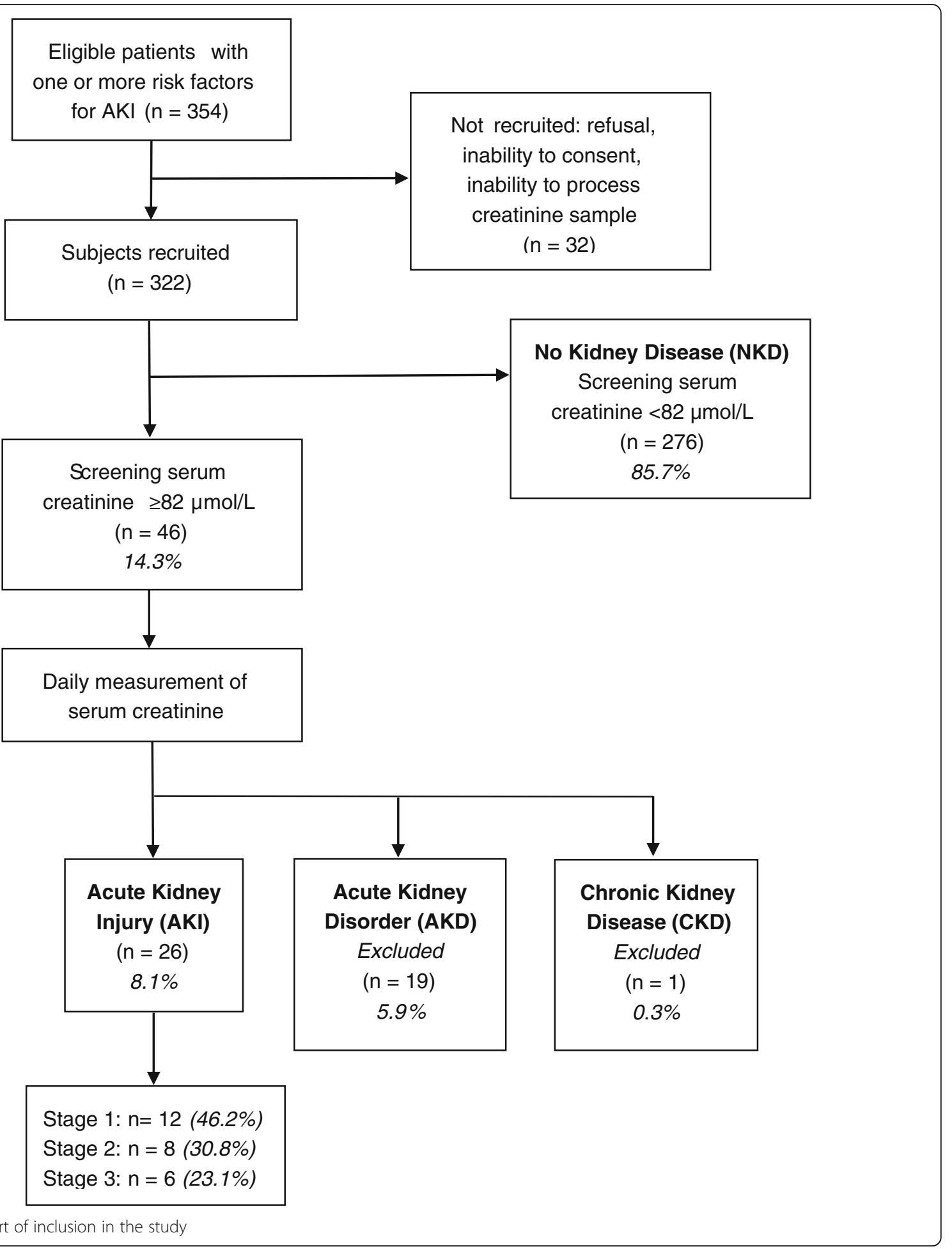

Fig. 1 Flow chart of inclusion in the study

(discharge SCr was 84, 98, and $106 \mu \mathrm{mol} / \mathrm{L}$ ); one had no renal recovery with discharge SCr $186 \mu \mathrm{mol} / \mathrm{L}$.

Maternal length of stay was assessed in 234/302 (77.5\%) cases: 16 mothers remained in hospital solely because their child was receiving neonatal care, 4 were transferred to private wards, 3 remained in hospital at the end of follow-up, and 45 were lost to follow-up. Median length of stay in women with NKD was 7 days (IQR 5 - 9) compared to 7 days (IQR 5 - 9) in women with AKI $(p=0.42)$.

Fetal outcomes were determined in 271/302 (89.7\%) cases, 1 was lost to follow-up, and 30 were recruited 
Table 2 Baseline data of women with acute kidney injury (AKI) and no kidney disease (NKD)

\begin{tabular}{llll}
\hline & NKD $(n=276)$ & AKI $(n=26)$ & $p$ value \\
\hline Age in years & $25(20-30)$ & $27(20-32)$ & 0.44 \\
Gravidity & $2(1-4)$ & $2(1-3)$ & 0.43 \\
Previous pregnancy loss & $70(25.4)$ & $5(19.2)$ & 0.49 \\
HIV positive & $33(12.0)$ & $1(3.9)$ & 0.43 \\
$\begin{array}{l}\text { Antiretroviral therapy (prior } \\
\text { to recruitment) }\end{array}$ & $29(10.5)$ & $1(3.9)$ & 0.28 \\
$\begin{array}{l}\text { Diabetes as co-morbidity } \\
\text { Any nephrotoxins }\end{array}$ (prior to & $1(0.36)$ & $0(0)$ & 0.76 \\
recruitment) & $99(36.0)$ & $8(30.8)$ & 0.60 \\
\hline
\end{tabular}

Figures shown are median (interquartile range) or frequency (\%)

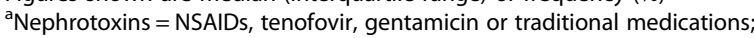
recorded if taken within 1 week of recruitment

antenatally and discharged prior to delivery. Fetal outcomes in patients with AKI and NKD are shown in Table 3. There were no statistically significant differences in any fetal outcome between AKI and NKD cohorts; median Apgar score at 1 min was lower in the AKI cohort (6) than in the NKD cohort (8), this approached statistical significance $(p=0.06)$.

Perinatal death occurred in 37/269 (13.8\%) cases overall, regardless of renal impairment. One of the four perinatal deaths in AKI patients was a stillbirth at 30 weeks (in a mother with preeclampsia and $\mathrm{APH}$, recruited at 3 days postpartum) suggesting fetal death likely preceded onset of AKI. The other three were early neonatal deaths: one mother with preeclampsia was recruited antenatally and delivered at 30 weeks; one delivered at 40 weeks and was recruited 2 days postpartum with endometritis; one delivered at 29 weeks with preeclampsia and sepsis and was recruited 2 days postpartum.

There was an association between preeclampsia spectrum (gestational hypertension, preeclampsia or eclampsia) and AKI. 20/164 (12.2\%) women recruited with preeclampsia spectrum had AKI; 6/138 (4.3\%) women without preeclampsia spectrum had AKI $(p=$ $0.015)$.

Table 3 Fetal outcomes in patients with acute kidney injury (AKI) no kidney disease (NKD)

\begin{tabular}{llll}
\hline & $\operatorname{NKD}(n=276)$ & AKI $(n=26)$ & $p$ value \\
\hline $\begin{array}{l}\text { Gestational age in weeks } \\
\text { (median; IQR) }\end{array}$ & $37.7(35.0-39.3)$ & $37.6(30.7-39.4)$ & 0.56 \\
Perinatal death (n; \%) & $33(13.6)$ & $4(15.4)$ & 0.8 \\
$\begin{array}{l}\text { Fetal weight in kg } \\
\text { (median; IQR) }\end{array}$ & $2.8(2.2-3.3)$ & $2.5(1.6-3.3)$ & 0.26 \\
Apgar score (median; IQR) & $8(5.0-8.0)$ & $6(4.0-8.0)$ & 0.06 \\
\hline
\end{tabular}

Figures shown are median (interquartile range) or frequency (\%)

\section{Discussion}

\section{Main findings}

To the best of our knowledge, this is the first study to prospectively investigate obstetric-related AKI outside intensive care and dialysis units in Sub-Saharan Africa. $8.1 \%$ of the cohort had AKI and the most common primary cause of AKI was preeclampsia spectrum, with a smaller contribution from sepsis and antepartum haemorrhage. No women with AKI died or required dialysis and the majority of AKI cases had complete renal recovery, even in stage 3 AKI. Fetal outcomes overall were poor with high rates of perinatal death. There were no statistically significant differences between AKI and NKD cohorts in either maternal or fetal outcomes.

We have identified ten publications investigating AKI in pregnancy from all of Africa since 1990 (Additional file 2). Three of these studies are from SSA; one investigates ICU patients [10], one investigates dialysis patients [9], and the other only includes patients with severe preeclampsia or eclampsia [11]. AKI prevalence amongst ICU patients varied from 6 to 34\% and the commonest causes of AKI reported were preeclampsiaeclampsia, haemorrhage and sepsis.

A recent population-wide study from Canada reported an AKI incidence rate of 2.68 per 10,000 deliveries, with aetiologies similar to the previous African studies [5]. This study found AKI in 26 (8.1\%) recruited women; given 2246 deliveries during the study period, we calculate the minimum incidence rate of AKI to be 116 per 10,000 deliveries in Malawi (over 40 times greater than the Canadian study). We have a novel cohort for SSA and cannot directly compare our AKI rate to that of previous ICU studies from the same region.

Whilst a recent review suggests sepsis and hypovolaemia are predominant causes of obstetric-related AKI in developing countries [1], our findings are consistent with those from other studies from Africa and match the patterns found in developed countries: preeclampsiaeclampsia underlies the majority of AKI. The association between preeclampsia spectrum at recruitment and AKI supports this. Table 4 demonstrates conditions contributing to the development of AKI in our cohort, as compared to data from Canada in 2007-10 [5]. Though the Canadian data define AKI based on clinician diagnosis as opposed to KDIGO criteria, there is an interesting comparison in AKI aetiology. Of note, there is a greater contribution of preeclampsia-eclampsia patients and a lesser contribution of postpartum haemorrhage to the development of AKI in our cohort.

Maternal outcomes in women with AKI were better than expected: no woman with AKI died or required dialysis. This may be accounted for by the quality of care in $\mathrm{QECH}$, a tertiary centre with doctors providing care, regular monitoring, and some access to blood tests and 
Table 4 Conditions contributing to the development of AKI in this study, compared to National Canadian data for 2007-10 [5]

\begin{tabular}{lll}
\hline Condition & $\begin{array}{l}\text { Malawi AKI cases } \\
(n=26) \\
\text { Current study }\end{array}$ & $\begin{array}{l}\text { Canadian AKI cases } \\
(n=290) \\
\text { Mehrabadi et al. [5] }\end{array}$ \\
\hline Antepartum haemorrhage & $4(15.4)$ & $13(4.5)$ \\
Postpartum haemorrhage & $2(7.7)$ & $91(31.4)$ \\
Gestational hypertension & $2(7.7)$ & $37(12.7)$ \\
Preeclampsia & $14(53.8)$ & $120(41.4)$ \\
Eclampsia & $4(15.4)$ & $10(3.5)$ \\
Sepsis & $4(15.4)$ & $36(12.4)$ \\
Cardiac failure & $1(3.8)$ & $21(7.2)$ \\
\hline
\end{tabular}

Multiple conditions are present in some patients. Canadian data from: Mehrabadi et al. [5]

medications. Renal impairment was detected early and women were managed jointly by nephrologists and obstetricians, a level of care which is not universal across SSA.

\section{Strengths and limitations}

This study reports data from a new population: obstetric ward admissions at high-risk of AKI in SSA. It complements previous studies looking at ICU and dialysis patients, providing data on less apparently unwell patients. Malawi provides a unique context to perform this study in SSA. Free access to healthcare means the sample selected and the care provided for these women was not confounded by their ability to pay for care. Moreover, whilst QECH is a tertiary healthcare facility, it also acts as the district hospital for Blantyre and the majority of admissions represent provision of secondary care to the local population, making the study more generalisable for the country as a whole.

The study was performed in a challenging context where access to investigations is limited. Practical issues (e.g. lack of urine measuring jugs, lack of catheter bags with graduations to accurately measure urine volume) made collecting data on urine output difficult. We were unable (logistically and financially) to measure $\mathrm{SCr}$ in all patients who delivered due to the number of deliveries that occur at this centre, and some of these patients may have had kidney injury leading us to underestimate true incidence of AKI. Similarly, we could not undertake serial SCr in every recruited woman, so used a single screening SCr to identify patients at risk of AKI, which we then targeted with serial $\mathrm{SCr}$ measurement. We chose to use a threshold which is 2 standard deviations above the mean in the third trimester, which may not be representative of this African population: as stated, some patients with screening creatinine $<82 \mu \mathrm{mol} / \mathrm{L}$ may have had AKI, especially those with lower BMI. Nevertheless this is a lower threshold than other comparable studies from SSA (a study from Cameroon defined renal failure as creatinine $>97 \mu \mathrm{mol} / \mathrm{L}$, a South African study as creatinine $>100 \mu \mathrm{mol} / \mathrm{L}[10,11])$ and highlights the challenges of diagnosing kidney disease in pregnancy more generally. Importantly, we only used this value to screen patients for kidney disease at recruitment, and AKI was confirmed on serial creatinine and urine output measurement thereafter and diagnosed according to the KDIGO criteria described. Serial SCr was not measured in women with a normal screening $\mathrm{SCr}$ and therefore hospital-acquired AKI was not assessed, although the majority of AKI in SSA is thought to be community acquired $[17,18]$.

Baseline data were recorded from the treating clinician's notes, which may have been inaccurate. It was not possible to obtain urine dipstick results from all recruited patients. The small study size and possible underestimation of AKI may mean some true associations between AKI and maternal-fetal outcomes were not identified. The study was performed outside malaria season, one of the commonest causes of AKI in Malawi [18], and the pattern of AKI may be different at other times of the year.

Care provided to study participants at QECH might not be considered representative of care across SSA. The presence of an obstetrician-staffed Obstetric High Dependency Unit is uncommon in SSA. Patients in the study benefited from measurement of serum creatinine, a test not routinely performed at $\mathrm{QECH}$ in obstetric patients outside of research studies, and rarely available in any patient elsewhere in Malawi. Those with elevated creatinine benefitted from management from specialist renal services. As such, the study is likely to underestimate AKI severity and overestimate renal recovery.

\section{Interpretation}

Overall, fetal outcomes were poor. The World Health Organisation reports the 2010 Malawi perinatal mortality rate to be $4 \%$ [19], which is significantly lower than the $13.8 \%$ in this study. Moreover, our narrower definition of perinatal mortality (only following neonates to discharge) likely underestimates the true value. Poor fetal outcomes might partly be accounted for by the recruitment of women with acute illness and preeclampsia itself may also play a role, given the majority of participants $(n=164 ; 54.3 \%)$ were recruited with this risk factor for AKI.

Preeclampsia is known to be a significant cause of obstetric AKI in developed and developing countries. A French ICU study reported 28\% (24/85) of admissions with eclampsia, HELLP or preeclampsia without HELLP developed AKI [20]. A study from Cameroon found $18.5 \%(10 / 54)$ of admissions with severe preeclampsia and eclampsia had AKI on day-1 post-partum [11]. Our study supports these findings, reporting a significant 
association between preeclampsia spectrum and AKI; $12.2 \%$ women recruited with preeclampsia spectrum had AKI. We feel this is of relevance to settings across SSA where preeclamptic patients present to healthcare facilities that often lack the capability to diagnose AKI. Indeed, given the inclusion of less severe cases of preeclampsia in our study compared to those cited above, the burden of preeclampsia-related AKI is relatively high.

Reasons for the prevalence of preeclampsia and its associated poor outcomes include: late antenatal booking visits; infrequent antenatal care; limited access to routine antenatal ultrasound; minimal use of aspirin prophylaxis; and delivery of antenatal care by non medically trained health care workers. Poor access to diagnostics for and interpretation of blood pressure and proteinuria meant most preeclampsia was diagnosed when symptomatic, thus advanced and more severe in nature. Additionally, recent data demonstrate pre-pregnancy recovered AKI is a risk factor for subsequent preeclampsia and poor fetal outcomes [21]. In a country where AKI is common and severe [18], previous recovered AKI may be a significant contributor to the preeclampsia burden; the interaction of recovered AKI, preeclampsia, and poor fetal outcomes provides an important avenue of future study.

We feel improving diagnosis and treatment of preeclampsia is key to preventing AKI, and moreover, improving fetal outcomes in Malawi. In a multigravid population, preventing repeated cycles of preeclampsiarelated AKI might in turn reduce the incidence of CKD. Better antenatal care is key to reducing the burden of preeclampsia and this will require increased provision of trained healthcare workers, better access to diagnostics including the development of simple point-of-care tests to detect kidney disease in rural settings [22], and population-level education regarding early presentation to antenatal care.

\section{Conclusions}

The incidence of obstetric AKI in Malawi is significantly greater than in developed countries and this study provides novel and important data for obstetricians, nephrologists and governments in the provision of healthcare. The majority of AKI was caused by preeclampsia, a potentially detectable and treatable disease which is also likely to underlie much of the perinatal mortality identified. Poor fetal outcomes reflect the challenges of antenatal and perinatal care in Malawi. Future work should investigate obstetric AKI and preeclampsia in health centres and district hospitals, where facilities and staffing are poorer, and address whether improved recognition and treatment of preeclampsia can reduce the incidence of AKI and improve fetal outcomes in this part of the world.

\section{Additional files}

Additional file 1: Definitions - Acute Kidney Injury, Acute Kidney Disorder, Chronic Kidney Disease, No Kidney Disease. Definitions from Kidney Disease: Improving Global Outcomes (KDIGO) criteria [15]. (DOCX 77 kb)

Additional file 2: Publications investigating AKI in pregnancy from Africa since 1990. Three studies from Sub-Saharan Africa are highlighted in grey. (DOCX 112 kb)

\section{Abbreviations \\ AKI: Acute Kidney Injury; CKD: Chronic Kidney Disease; NKD: No kidney disease; SCr: Serum creatinine; SSA: Sub-Saharan Africa}

\section{Acknowledgements}

The authors are grateful to Edward Senga and Felix Dzumani at the Malaw College of Medicine for their assistance with laboratory services, and David Williams at University College London for his advice on interpretation of results.

\section{Funding}

The study was funded by The Royal Society of Tropical Medicine and Hygiene (RE) and The Royal College of Obstetricians and Gynaecologists (WC - Eden Travelling Fellowship). The role of both of these was the provision of funding only.

\section{Availability of data and materials}

The datasets analysed during the current study are available from the corresponding author on reasonable request.

\section{Authors' contributions}

WC, UH, AC, AM, GD and RE designed the study. WC, UH, AC, CM and PM acquired data. WC, RE, and GD analysed the data and performed statistical analyses. WC, GD and RE drafted the manuscript; UH, AC, CM, AM, PM critically appraised its content. All authors have revised the manuscript, have approved the final version, and take public responsibility for its content. All authors agree to be accountable for all aspects of the work.

\section{Ethics approval and consent to participate}

The study was approved by the University of Malawi, College of Medicine Research Ethics Committee (protocol number P.11/14/1660). Written informed consent was obtained from all subjects before participation.

Consent for publication

Not applicable.

Competing interests

The authors declare that they have no competing interests.

\section{Publisher's Note}

Springer Nature remains neutral with regard to jurisdictional claims in published maps and institutional affiliations.

\section{Author details}

'Department of Internal Medicine, Queen Elizabeth Central Hospital, Blantyre, Malawi. ${ }^{2}$ Department of Obstetrics and Gynaecology, Royal Berkshire Hospital, Craven Road, Reading RG1 5AN, UK. ${ }^{3}$ Department of Obstetrics and Gynaecology, Queen Elizabeth Central Hospital, Blantyre, Malawi. ${ }^{4}$ University College London Centre for Nephrology, Royal Free Hospital, Pond Street, London NW3 2QG, UK. ${ }^{5}$ Department of Nephrology, Bart's Health NHS Trust, London, UK.

Received: 28 March 2017 Accepted: 21 January 2018

Published online: 02 February 2018

\section{References}

1. Acharya A, Santos J, Linde B, Anis K. Acute kidney injury in pregnancycurrent status. Adv Chronic Kidney Dis. 2013;20:215-22. Elsevier Ltd.

2. Gammill HS, Jeyabalan A. Acute renal failure in pregnancy. Crit Care Med. 2005;33:S372-84. United States. 
3. Jha V, Parameswaran S. Community-acquired acute kidney injury in tropical countries. Nat Rev Nephrol. 2013;9:278-90. Nature Publishing Group.

4. Stratta P, Besso L, Canavese C, Grill A, Todros T, Benedetto C, et al. Is pregnancy-related acute renal failure a disappearing clinical entity? Ren Fail. 1996;18:575-84. United States.

5. Mehrabadi A, Liu S, Bartholomew S, Hutcheon JA, Magee LA, Kramer MS, et al. Hypertensive disorders of pregnancy and the recent increase in obstetric acute renal failure in Canada: population based retrospective cohort study. BMJ. 2014;349:94731.

6. Arrayhani M, El Youbi R, Sqalli T. Pregnancy-related acute kidney injury: experience of the nephrology unit at the university hospital of fez, morocco. ISRN Nephrol. 2013;2013:1-5.

7. Mehta RL, Cerda J, Burdmann EA, Tonelli M, Garcia-Garcia G, Jha V, et al. International society of Nephrology's 0 by25 initiative for acute kidney injury (zero preventable deaths by 2025): a human rights case for nephrology. Lancet. 2015;385:2616-43. England.

8. World Health Organisation. World health organisation. Factsheet number 348 [internet]. 2014 [cited 2015 Nov 10]. Available from: http://www.who. int/mediacentre/factsheets/fs348/en/.

9. Randeree IG, Czarnocki A, Moodley J, Seedat YK, Naiker IP. Acute renal failure in pregnancy in South Africa. Ren Fail. 1995;17:147-53.

10. Drakeley AJ, Le Roux PA, Anthony J, Penny J. Acute renal failure complicating severe preeclampsia requiring admission to an obstetric intensive care unit. Am J Obstet Gynecol. 2002;186:253-6.

11. Kaze FF, Njukeng FA, Kengne A-P, Ashuntantang G, Mbu R, Halle MP, et al. Post-partum trend in blood pressure levels, renal function and proteinuria in women with severe preeclampsia and eclampsia in sub-Saharan Africa: a 6-months cohort study. BMC Pregnancy Childbirth. 2014;14:134.

12. The World Bank. World Development Indicators: Reproductive health [Internet]. 2014 [cited 2016 May 30]. p. Table 2.17. Available from: http://wdi. worldbank.org/table/2.17.

13. Tessema GA, Tekeste A, Ayele TA. Preeclampsia and associated factors among pregnant women attending antenatal care in Dessie referral hospital, Northeast Ethiopia: a hospital-based study. BMC Pregnancy Childbirth. 2015;15:73.

14. Williams D, Davison J. Chronic kidney disease in pregnancy. BMJ. 2008;336: 211-5. England.

15. Kidney Disease: Improving Global Outcomes (KDIGO) Acute Kidney Injury Work Group. KDIGO clinical practice guideline for acute kidney injury. Kidney Int. 2012;2:1-138.

16. Apgar V. A proposal for a new method of evaluation of the newborn infant. Originally published in July 1953 , volume 32 , pages $250-259$. Anesth Analg. 2015;120:1056-9. United States.

17. Olowu WA, Niang A, Osafo C, Ashuntantang G, Arogundade FA, Porter J, et al. Outcomes of acute kidney injury in children and adults in sub-Saharan Africa: a systematic review. Lancet Glob Health. 2016;4:e242-50. England.

18. Evans RDR, Hemmila U, Craik A, Mtekateka M, Hamilton F, Kawale Z, et al. Incidence, aetiology and outcome of community-acquired acute kidney injury in medical admissions in Malawi. BMC Nephrol. 2017;18:21. England.

19. World Health Organisation. Malawi maternal and Perinatal health profile [internet]. 2015 [cited 2016 May 30]. Available from: http://www.who.int/ maternal_child_adolescent/epidemiology/en/.

20. Jonard M, Ducloy-Bouthors A-S, Boyle E, Aucourt M, Gasan G, Jourdain M, et al. Postpartum acute renal failure: a multicenter study of risk factors in patients admitted to ICU. Ann Intensive Care. 2014;4:36.

21. Tangren JS, Powe CE, Ankers E, Ecker J, Bramham K, Hladunewich MA, et al. Pregnancy outcomes after clinical recovery from AKI. J Am Soc Nephrol JASN. 2017;28:1566-74.

22. Evans R, Calice-Silva V, Raimann JG, Hemmila U, Craik A, Mtekateka M, et al. Diagnostic performance of a saliva urea nitrogen dipstick to detect kidney disease in Malawi. Kidney Int Rep. 2017;2:219-27. Elsevier.

23. Roberts JM, August PA, Bakris $G$, Barton JR, Bernstein IM, Druzin M, et al. Hypertension in pregnancy. Report of the American College of Obstetricians and Gynecologists' Task Force on Hypertension in Pregnancy. Obstet Gynecol. 2013;122:1122-31. United States.

\section{Submit your next manuscript to BioMed Central and we will help you at every step:}

- We accept pre-submission inquiries

- Our selector tool helps you to find the most relevant journal

- We provide round the clock customer support

- Convenient online submission

- Thorough peer review

- Inclusion in PubMed and all major indexing services

- Maximum visibility for your research

Submit your manuscript at www.biomedcentral.com/submit
Biomed Central 